\title{
DIE GRUNDLAGEN DER SOZIALEN
}

MARKTWIRTSCHAFT

von

Prof. (em.) Dr. Hans-Rimbert Hemmer 


\section{Gliederung:}

1. Die Wirtschaftsordnung als Rahmenbedingung wirtschaftlicher Entwicklung

2. Der Grundgedanke der Sozialen Marktwirtschaft

a) Grundposition 1: Die Soziale Marktwirtschaft ist eine Marktwirtschaft

b) Grundposition 2: Die Soziale Marktwirtschaft verknüpft die marktwirtschaftlicher Leistung mit einem sozialen Ausgleich

c) Grundposition 3: Die Beziehungen zwischen marktwirtschaftlicher Leistung und sozialem Ausgleich werden vom Subsidiaritätsprinzip bestimmt

d) Die Wortverbindung „Soziale Marktwirtschaft“ - ein

Widerspruch?

3. Wirtschafts-und sozialpolitische Ziele der Sozialen Marktwirtschaft

a) „Oberste“ Ziele in einer demokratischen Gesellschaftsordnung 8

b) Gesamtwirtschaftliche Ziele 10

c) Soziale Ziele

4. Aufgaben des Staates in der Sozialen Marktwirtschaft: Grundlagen

a) Die konstituierenden Prinzipien Euckens

b) Ordnungspolitik und/oder Prozesspolitik als Handlungsvorgaben staatlicher Interventionen

5. Aufgaben des Staates in der Sozialen Marktwirtschaft:

Die zentralen Politikfelder

a) Die Schaffung der erforderlichen Rahmenbedingungen 15

b) Die Bereitstellung öffentlicher Güter

c) Die Wettbewerbspolitik als Politikbereich zur Realisierung der Marktwirtschaftskomponente der Sozialen Marktwirtschaft

d) Die Sozialpolitik als Politikbereich zur Realisierung der Sozialkomponente der Sozialen Marktwirtschaft

6. Zusammenfassung: Die Soziale Marktwirtschaft als ordnungspolitisches Konzept 


\section{Die Wirtschaftsordnung als Rahmenbedingung wirtschaftlicher Entwicklung}

(1) Alle modernen Volkswirtschaften kennzeichnen sich durch einen hohen Grad an Arbeitsteilung und damit eine intensive Spezialisierung der einzelnen Wirtschaftssubjekte. Um die Funktionsfähigkeit dieser arbeitsteiligen Volkswirtschaften sicherzustellen, müssen die verschiedenen ökonomischen Aktivitäten innerhalb einer Volkswirtschaft aber koordiniert werden. Soll ein chaotisches Nebeneinander einzelwirtschaftlicher Aktionen vermieden werden, sind Regeln, Normen und Institutionen erforderlich, die von allen Wirtschaftssubjekten akzeptiert werden müssen - die Wirtschaftsordnungen. Unter dem Begriff „Wirtschaftsordnungen“ fasst man nämlich alle Regeln (d.h. Organisationsprinzipien, Normen, Lenkungs- und Entscheidungsmechanismen, Einrichtungen und Verhaltensweisen) für das wirtschaftliche und wirtschaftspolitische Handeln zusammen, denen die einzelnen Mitglieder der Gesellschaft unterworfen sind und die ihre wirtschaftlichen Aktivitäten steuern.

(2) Für die konkrete Ausgestaltung von Wirtschaftsordnungen ist es von entscheidender Bedeutung, welche Steuerungsmechanismen für die ökonomischen Aktivitäten der einzelnen Gesellschaftsmitglieder zugrunde gelegt werden. In der älteren ordnungstheoretischen Literatur der 30er und 40er Jahre des vergangenen Jahrhunderts, in denen die Konzeption der Sozialen Marktwirtschaft entwickelt und die Weichen für ihre Implementierung gestellt wurden, hat man in vereinfachter Form zwei Steuerungsmechanismen unterschieden:

a) Werden die ökonomischen Aktivitäten dezentral über Märkte gesteuert, d.h. die Wirtschaftssubjekte orientieren sich an den Bedingungen, die sie auf Märkten vorfinden, so spricht man von einer marktwirtschaftlichen Steuerung.

b) Werden die ökonomischen Aktivitäten zentral vom Staat vorgegeben, d. h. die Wirtschaftssubjekte setzen zentrale Verwaltungsvorgaben um, so spricht man von einer zentralverwaltungswirtschaftlichen Steuerung.

(3) Die einzelnen realen Wirtschaftsordnungen unterscheiden sich danach, in welchem Mischungsverhältnis diese beiden Steuerungsmechanismen zueinander stehen bzw. verwendet werden. Vom empirischen Befund her hat sich der marktwirtschaftliche Steuerungsmechanismus für viele Bereiche wirtschaftlicher Aktivitäten als überlegen erwiesen. Der weltweite Zusammenbruch des das Zentralplanungsprinzip bevorzugenden realen Sozialismus Ende der 80er/Anfang der 90er Jahre und die in vielen Ländern feststellbare Tendenz zu Reformen, die eine stärkere Berücksichtigung marktwirtschaftlicher Steuerungsmechanismen zum Gegenstand haben, unterstreichen dieses Ergebnis. 


\section{Der Grundgedanke der Sozialen Marktwirtschaft}

\section{a) Grundposition 1: Die Soziale Marktwirtschaft ist eine Marktwirtschaft}

(1) Die in der Bundesrepublik Deutschland nach dem Ende des II. Weltkriegs eingeführte Wirtschaftsordnung der „Sozialen Marktwirtschaft“ basiert auf den grundlegenden Arbeiten von Alfred Müller-Armack und Ludwig Erhard unter Rückgriff auf viele Vorarbeiten der sogenannten „Freiburger Schule“ bzw. des Ordoliberalismus, vor allem von Walter Eucken, Franz Böhm und Wilhelm Röpke.

(2) Die negativen Erfahrungen, die vor allem Ende des 19./Anfang des 20. Jahrhunderts mit interventionistischen Mischsystemen gemacht wurden, die unter dem Eindruck von Depressionen und Massenarbeitslosigkeit entstanden sind, ohne zu deren Beseitigung beigetragen zu haben, ließen bei den Vätern der Sozialen Marktwirtschaft die Einsicht entstehen, daß Wirtschaftspolitik ohne eine klare Entscheidung für ein Koordinationsprinzip - Markt oder Befehl - nicht erfolgreich sein kann. Angesichts der Erfahrungen während der Hitler-Diktatur sowie aufgrund eigener philosophischer Grundpositionen fiel diese Entscheidung zugunsten jenes Koordinationsmechanismus aus, der ein Maximum an individueller Freiheit - dem höchsten Wert der Gemeinschaft - sichert: der Markt. Insofern ist die Soziale Marktwirtschaft eindeutig eine Marktwirtschaft.

(3) Mit ihrem individualistischen Menschenbild stützten sich die „Väter“ der Sozialen Marktwirtschaft auf die Gedanken der Aufklärung, in deren Mittelpunkt das Individuum steht. Dieses ist nach Auffassung der Aufklärer ein vernunftbegabtes Wesen, das die Fähigkeit zu und ein Recht auf freie Entfaltung hat. In der Ausnutzung seiner ihm eingeräumten Freiheit, die in allen ihren Aspekten, den geistigen, politischen und materiellen, zu verstehen ist, kann der einzelne Mensch durch den Einsatz seiner Leistung die persönlichen, selbstgesteckten Ziele zu verwirklichen suchen.

Damit die freie Entfaltung der Individuen allerdings nicht zu einem „Krieg aller gegen alle“ ausartet, muß in der Gesellschaft eine harmonische Ordnung herrschen, die sich dadurch auszeichnet, daß der einzelne seine Freiheit nicht auf Kosten seiner Mitbürger ausnützt; und diese Ordnung bietet (nur) eine sozial fokussierte Wettbewerbswirtschaft. Die Wirkungsweise des Marktmechanismus muss sich deshalb innerhalb von Rahmenbedingungen entfalten, die so konstruiert sind, daß sie weitgehend zu sozial akzeptablen Ergebnissen des Marktes führen.

(4) Dabei ist auch zu berïcksichtigen, dass es letztlich unmöglich (und dort, wo technisch möglich, ökonomisch nicht immer zweckmäßig) ist, eine Volkswirtschaft ausschließlich durch den Marktmechanismus zu steuern: Einige Bereiche sind überhaupt nicht über den Marktmechanismus steuerbar (Fall des Marktversagens), in anderen Fällen würde die Steuerung über den Markt zu Kosten führen, die wesentlich höher sind als die erzielbaren Vorteile für die Gesellschaft (Fall der Marktineffizienz). In einem dritten Bereich würden sich bei ausschließlicher Zugrundelegung des marktwirtschaftlichen Steuerungsprinzips zumindest in Teilbereichen Ergebnisse einstellen, welche nicht die tatsächlichen 
gesamtwirtschaftlichen Knappheitsverhältnisse der Faktoren und Güter widerspiegeln oder aber zentralen gesellschaftlichen Wertvorstellungen widersprechen (Fall der Marktablehnung). Daraus resultieren aber nicht - wie noch von der älteren Ordnungstheorie gefolgert wurde - zwingend Staatsinterventionen. Vielmehr sind sowohl privatwirtschaftlich getragene Koordinationsprozesse innerhalb von Hierarchien oder Netzwerken als auch staatliche Korrekturmaßnahmen bis hin zur Einrichtung von staatlich kontrollierten Wirtschaftsbereichen erforderlich. Es muss für den Einzelfall entschieden werden, welches Steuerungsprinzip jeweils die beste Lösung darstellt. Plakativ ausgedrückt: Es geht nicht um „Markt oder Staat“, sondern um die optimale Kombination von „Markt und Staat" unter gleichzeitiger Berücksichtigung der Steuerungspotentiale privatwirtschaftlich getragener Hierarchien und Netzwerke.

(5) Als ein besonders wichtiger theoretischer Vordenker der Sozialen Marktwirtschaft gilt Walter Eucken. Eucken war konsequenter Befürworter einer dezentralen Steuerung der Wirtschaft durch Markt und Wettbewerb. Er wandte sich aber entschieden gegen eine Rückkehr zur freien Marktwirtschaft, die vor 1933, dem Jahr der Machtergreifung des Nationalsozialismus, in Deutschland bestanden hatte. Sein Haupteinwand gegen die traditionelle liberale Politik des Laissez-faire war, dass diese uneingeschränkte Vertragsfreiheit einräumte, auch die Freiheit zum Abschluss von Verträgen, durch die die Unternehmen den Wettbewerb zu ihrem eigenen Vorteil und zum Nachteil der Konsumenten einschränken. Dies hatte in der Vergangenheit in Deutschland zur Bildung mächtiger Kartelle geführt, die weite Teile der Wirtschaft beherrschten. In der Einschränkung des Wettbewerbs durch Kartelle, in der dadurch bedingten Ausschaltung der marktwirtschaftlichen Lenkung durch den Preismechanismus und in der unzureichenden Anpassungsfähigkeit der Kartelle sah Eucken eine der Hauptursachen für die wirtschaftliche Krise, die Deutschland zu Beginn der 30er Jahre heimgesucht hatte.

(6) Gegen die traditionelle liberale Vorstellung von einem Staat, der sich auf die Aufrechterhaltung von Sicherheit und Ordnung zu beschränken, im übrigen aber die Wirtschaft sich selbst zu überlassen habe, setzte Eucken die Forderung nach einem starken Staat, der die Wirtschaftsordnung zielbewusst gestaltet, sich erfolgreich gegen die inzwischen unermesslich erscheinenden Forderungen von Lobbyisten und partikularen Interessengruppen zur Wehr setzen kann, Regeln für den Wettbewerb durchsetzt und damit die Entstehung wirtschaftlicher Machtpositionen verhindert. Diese Forderung nach einem starken Staat hat im Prinzip bereits Rüstow im Jahr 1932 erhoben: „Eben deshalb fordert das, was ich vorschlage, einen starken Staat. Einen Staat, der über den Gruppen, über den Interessen steht. Einen Staat, der sich aus der Verstrickung der Wirtschaftsinteressen, wenn er in sie hineingeraten ist, wieder herauslöst. Und gerade dieses sich Besinnen und sich Zurückziehen des Staates auf sich selbst, diese Selbstbeschränkung als Grundlage der Selbstbehauptung ist Voraussetzung und Ausdruck seiner Unabhängigkeit und Stärke.“ 


\section{b) Grundposition 2: Die Soziale Marktwirtschaft verknüpft die marktwirtschaftlicher Leistung mit einem sozialen Ausgleich}

(1) Nach Aufffassung ihrer Vordenker verbindet die Soziale Marktwirtschaft auf der Basis der Wettbewerbswirtschaft die freie Initiative mit einem gerade durch die marktwirtschaftliche Leistung gesicherten sozialen Fortschritt. Die Soziale Marktwirtschaft ist also keine freie Marktwirtschaft, die ausschließlich durch Wettbewerb und Individualismus bestimmt wird. Alfred Müller-Armack hat es als Sinn der Sozialen Marktwirtschaft bezeichnet, ,das Prinzip der Freiheit auf dem Markte mit dem des Sozialen Ausgleichs zu verbinden“.

(2) Für die Begründer der Sozialen Marktwirtschaft war und ist es auch aus heutiger Sicht falsch obwohl dies vor allem von sozialistischer Seite immer wieder geleugnet wird -, die Marktwirtschaft und das Soziale als etwas Getrenntes oder Gegensätzliches bzw. den sozialen Ausgleich ausschließlich als das Ergebnis staatlicher Umverteilung zu sehen. Vielmehr stehen Eigenverantwortung und private Absicherung eindeutig an erster Stelle. Die Gemeinschaft soll aber auch denjenigen ein menschenwürdiges Leben garantieren, die Hilfe brauchen: den weniger Leistungsfähigen, Alten, Kranken, Behinderten. Und sie soll bei der Eigenvorsorge für die großen Lebensrisiken helfen. Insofern soll mit der Wirtschaftsordnung der Sozialen Marktwirtschaft eine Synthese zwischen dem Ziel individueller Freiheit und dem der sozialen Bindung menschlicher Verhaltensweisen gefunden werden; soziale Zielsetzungen (,soziale Sicherung“, „soziale Gerechtigkeit“, „sozialer Fortschritt“) sollen auf der Basis freier Initiative des Individuums, also bei möglichst großen individuellen Freiheitsspielräumen, verwirklicht werden. Diese Verknüpfung von individueller Freiheit und sozialem Ausgleich kann aber nur bei einem im Grundsatz marktwirtschaftlichen System erfolgen, da nur hier individuelle Freiheit möglich ist. Entsprechend wichtig für das Konzept der Sozialen Marktwirtschaft ist es deshalb, den Wettbewerb und die durch ihn geschaffenen Spielräume autonomer Planung und Entscheidung so zu sichern, daß er ein hohes Maß an individuellen Initiativen und Aktivitäten freisetzt, um die ökonomische Potenz einer Gesellschaft zu stärken und so ihre dauerhafte wirtschaftliche Leistungsfähigkeit zu sichern. Wirtschaftliche Leistungsfähigkeit gilt wiederum als Voraussetzung dafür, soziale Zielsetzungen zu realisieren. Insofern sind persönliche Freiheit, soziale Gerechtigkeit und ökonomische Leistungsfähigkeit wesentliche und untrennbar miteinander verbundene Bestandteile des Konzepts der Sozialen Markwirtschaft.

(3) Für die Verfechter der Sozialen Marktwirtschaft bestand das zentrale Gestaltungsproblem darin, eine Wirtschaftsordnung zu konzipieren, welche im Sinne der hier vorgebrachten Überlegungen Leistungswettbewerb und soziale Sicherung integriert, um durch ihr gemeinsames Wirken das immer aktuelle sozio-ökonomische Konfliktpotential in einer Gesellschaft zu mindern. Entsprechend dieser Intention wird zwar der Leistungswettbewerb als wirtschaftliche Grundlage sozialer Sicherheit und sozialen Fortschritts gefördert und auch prinzipiell als Regulator der Einkommensverteilung akzeptiert; dem Staat wird jedoch gleichzeitig die Aufgabe einer umfassenden Sozialpolitik 
zugeschrieben. Er soll durch eine aktive Politik der Einkommens- und Vermögensumverteilung das soziale Gefälle in der Gesellschaft mindern (z.B. Eigentumsbildung in Arbeitnehmerhand) sowie unter Wahrung des Freiheitspostulats einen tendenziellen Ausgleich von Start-, Einkommens-, Vermögensund Belastungsunterschieden herbeiführen. Diejenigen, die am Leistungswettbewerb nicht, noch nicht oder nicht mehr teilnehmen können und deshalb auch kein Markteinkommen erhalten, sollen nicht nur existenziell abgesichert werden, sondern auch am ökonomischen Fortschritt partizipieren. Darüber hinaus ist es Aufgabe der speziellen Sozialpolitik des Staates, durch Rechtsvorschriften den Menschen als Träger von Arbeitsleistungen zu schützen (z.B. Schutz der Gesundheit und des Rechts auf Persönlichkeitsentfaltung). Insofern gilt die „Soziale Marktwirtschaft“ als „,irenische Formel“1: Die internen Konflikte der Gesellschaft sind als Realität hinzunehmen und über die Soziale Marktwirtschaft zu lösen.

\section{c) Grundposition 3: Die Beziehungen zwischen marktwirtschaftlicher Leistung und sozialem Ausgleich werden vom Subsidiaritätsprinzip bestimmt}

(1) Bei der Lösung ist - und hierin drückt sich wieder der hohe Stellenwert des Freiheitspostulats aus - dem Subsidiaritätsprinzip Rechnung zu tragen, das der Eigeninitiative des Individuums oder Gruppen von Individuen den Vorzug vor dem Staat gibt.

Das Subsidiaritätsprinzip:

Das Subsidiaritätsprinzip beinhaltet die Grundeinstellung, daß es gegen die Gerechtigkeit verstoße, wenn für das, was die kleineren und untergeordneten Gemeinwesen leisten und „zum guten Ende führen“ können, die übergeordnete Gemeinschaft in Anspruch genommen wird. Innerhalb dieses Grundsatzes lassen sich dann zwei Versionen des Subsidiaritätsprinzips ausmachen:

a) Die negative Version besagt, daß die übergeordnete Gemeinschaft erst dann tätig werden darf, wenn die zu lösende Aufgabe die Kräfte der untergeordneten Gemeinschaft übersteigt. Damit bezieht die negative Version Stellung gegen einen übermäßigen Staatsinterventionismus.

b) In der positiven Version soll die übergeordnete Gemeinschaft ihren Mitgliedern Hilfe und Beistand leisten, um sie in die Lage zu versetzen, möglichst viele Aufgaben selbst zu erledigen.

(2) Entsprechend dem Subsidiaritätsprinzip sollte sich die Zuordnung einzelner Wirtschaftsbereiche zu den Koordinationsmechanismen Markt, Organisation oder Staat an der Funktionsfähigkeit des dezentralen Lenkungsmechanismus ausrichten. Dieses Prinzip führt dann $\mathrm{zu}$ den folgenden Konsequenzen:

a) Man sollte die wirtschaftlichen Entscheidungen einem marktwirtschaftlichen Koordinationsmechanismus überlassen, soweit die Orientierung der Marktteilnehmer an ihren individuellen

1 griechisch: eirene $=$ Friede. 
Zielvorstellungen zu gesamtwirtschaftlich oder - bei metaökonomischer Betrachtung gesellschaftlich erwünschten Resultaten führt. Organisationsinterne Koordinierungen sind der Marktsteuerung dabei gleichgestellt. Offen bleibt dabei, wer feststellen darf, unter wann ein Resultat „gesamtwirtschaftlich erwünscht“ ist.

b) Eine staatliche Beeinflussung privatwirtschaftlicher Abläufe sollte dort vorgenommen werden, wo die ausschließliche Befolgung der individuellen Zielvorstellungen nicht zu gesamtwirtschaftlich bzw. gesellschaftlich erwünschten Resultaten führt und der Staat in der Lage ist, den Konflikt zwischen einzelwirtschaftlichen und gesamtwirtschaftlichen bzw. gesellschaftlichen Zielen durch entsprechende Maßnahmen aufzulösen. Die Wahl der Maßnahmen muß dabei unter dem Aspekt erfolgen, daß aus gesamtwirtschaftlicher bzw. gesellschaftlicher Sicht ihre Kosten geringer sind als die erzielbaren Ergebnisverbesserungen. Dabei sollte vorrangig versucht werden, die für die privatwirtschaftlichen Aktivitäten maßgeblichen institutionellen Rahmenbedingungen (bspw. das Ausmaß der Verwertbarkeit von Informationen) so zu verändern, daß aus dem privaten Handeln gesamtwirtschaftlich bessere Ergebnisse als zuvor resultieren. Dies setzt nicht zuletzt die Fähigkeit der staatlichen Instanzen voraus, den Druck organisierter Interessengruppen, die partielle Privilegien auf Kosten der Gesellschaft erreichen bzw. sichern wollen (rent-seeking), abzublocken.

c) Die völlige staatliche Lenkung ohne Zwischenschaltung privatwirtschaftlicher Aktivitäten empfiehlt sich lediglich für jene Fälle, bei denen die Konfliktauflösung zwischen einzel- und gesamtwirtschaftlichen Zielvorstellungen nur mit unverhältnismäßig hohen (verglichen mit der Ergebnisänderung) Kosten oder überhaupt nicht erreicht werden kann.

Eine grundsätzliche Zuordnung konkreter Wirtschaftsbereiche zu einer dieser drei Gruppen ist allerdings nicht möglich: Hier müssen stets die Bedingungen des Einzelfalls berücksichtigt werden. Aber in allen drei Fällen verbleibt der Staat weiterhin in seiner grundlegenden Verantwortung, die Voraussetzungen für die Funktionsfähigkeit und Selbststeuerung privatwirtschaftlicher Koordinationsmechanismen zu schaffen. Die Umsetzung dieser Voraussetzungen wird damit zur Grundlage einer Sozialen Marktwirtschaft. Ihre Konkretisierung wird aber in jedem Einzelfall anders aussehen müssen; Patentrezepte für eine solche Soziale Marktwirtschaft gibt es jedenfalls nicht.

(3) Aus dieser Perspektive finden in der irenischen Formel dann bspw. auch Gewerkschaften, Mitbestimmung („Mitsprache in sozialen Fragen“), Gewinnbeteiligung, Verbraucherschutz sowie viele Kooperationsformen (z.B. Genossenschaften und Verbände) ihren Platz. In dieser hervorgehobenen Position des Subsidiaritätsprinzips wird auch der zentrale Unterschied der Sozialen Marktwirtschaft gegenüber dem stärker von sozialistischem Gedankengut geprägten Wohlfahrts- bzw. Sozialstaat deutlich: Bei ihm hat das Solidaritätsprinzip mit seiner impliziten Umverteilungsideologie eindeutig Vorrang gegenüber dem stärker leistungsbezogenen Subsidiaritätsprinzip; in der Sozialen 
Marktwirtschaft sind beide Prinzipien hingegen gleichrangig. Mehr noch: Soziale Korrekturen müssen von ihrer Größenordnung und ihrer strukturellen Wirkung her stets so dimensioniert sein, dass sie nicht das Marktprinzip mit seiner inhärenten Leistungsorientierung unterminieren. Sozialkorrekturen sind also nur mit einer Sozialen Marktwirtschaft vereinbar, solange sie sich nicht destruktiv auf die Leistungsfähigkeit und -bereitschaft der Wirtschaft und Gesellschaft auswirken. Dazu müssen sie selbst wieder dem Subsidiaritätsprinzip genüge tun.

\section{d) Die Wortverbindung ,Soziale Marktwirtschaft“ - ein Widerspruch?}

(1) Die Wortverbindung „Soziale Marktwirtschaft“ ist vielfach als widersprüchlich empfunden worden, weil in dem individualistischen und das Eigeninteresse betonenden Wettbewerbssystem „soziale“ (oder auf das Gemeinwohl zielende) Verhaltensweisen der wirtschaftlichen Menschen nicht ausdrücklich vorgesehen sind. Dieser Vorwurf verkennt aber das Wesen der Sozialen Marktwirtschaft; das „soziale“ muß hier nämlich in ihrer Gesamtkonzeption einschließlich der Aufgabenfelder des Staates erblickt werden. Von der Konzeption der Sozialen Marktwirtschaft her verdienen vor allem die „Freiheiten“, die das System gewährt, das Attribut „,sozial“. Die Dimensionen dieser ökonomischen Freiheiten sind zu sehen

* in der freien Konsumwahl, d.h. in der unbeeinflußten (,souveränen“) Entscheidung des Konsumenten, dieses oder jenes Gut zu kaufen bzw. diese oder jene Dienstleistung in Anspruch zu nehmen;

* $\quad$ in der freien Berufs- und Arbeitsplatzwahl. Dies schließt die freie Wahl der Berufsausbildung mit ein;

* schließlich in der freien Wahl der Produktion bzw. der Art der Produktionsfaktorkombinationen.

(2) Die Konsumentensouveränität und in deren Gefolge die Orientierung der Produktion am Verbrauch dürfen allein deswegen als soziale Leistung angesehen werden, weil die alle sozialen Schichten umfassenden und in ihrer Machtposition überdies schwach gesicherten Konsumenten nur in einem marktwirtschaftlichen System die Wirtschaft nach ihren Bedürfnissen lenken können. In Zentralverwaltungswirtschaften dagegen besteht die ständige Gefahr, daß die Güterströme nach den unerfindlichen Ratschlüssen der anonymen und übermächtigen Planungsbehörden an den Wünschen der Konsumenten vorbeigelenkt werden. Aus diesem Grunde läuft ja auch die Soziale Marktwirtschaft entsprechend den Vorstellungen ihrer Befürworter nach den Regeln der Marktwirtschaft ab. Sie ist allerdings zusätzlich mit sozialen Sicherungen und Ergänzungen versehen und präsentiert sich von daher als eine Wettbewerbsordnung, die die freie Initiative mit einem gerade durch die marktwirtschaftliche Leistung gesicherten sozialen Fortschritt in Einklang zu bringen versucht.

(3) Die meisten Vertreter des Ordoliberalismus waren überzeugt, dass sie mit diesem Ansatz ein spezifisch deutsches Konzept der Sozialen Marktwirtschaft entworfen hatten - vor dem Hintergrund 
der Erfahrung mit der ordnungspolitischen Orientierungslosigkeit der Weimarer Republik und vor allem der totalitären Katastrophe des Nationalsozialismus. Nach gut einem halben Jahrhundert Nachkriegsgeschichte kann allerdings hinterfragt werden, ob die Unterschiede zwischen den marktwirtschaftlichen Modellen der kontinentaleuropäischen Länder wirklich so groß sind, wie es der Blick auf ihre jeweiligen philosophischen Grundlagen vermuten lassen könnte. Tatsächlich hat es durchaus Sinn, von einem europäischen - und nicht einem deutschen - Modell des gezähmten Kapitalismus zu sprechen. Die konstituierenden Elemente dieses Modells sind eine im Grundsatz marktwirtschaftliche Rechts- und Wettbewerbsordnung mit einem gut ausgebauten Sozialstaat und zum Teil reguliertem Arbeitsmarkt sowie mehr oder weniger stark zentralisierte Tarifverhandlungen. Die Unterschiede zwischen den kontinentaleuropäischen Ländern sind dabei historisch bedingt und eher graduell: zum Beispiel in Frankreich ein stärkerer Staatseinfluss bei schwächeren Gewerkschaften, in Deutschland umgekehrt.

\section{Wirtschafts- und sozialpolitische Ziele der Sozialen Marktwirtschaft}

\section{a) „Oberste“ Ziele in einer demokratischen Gesellschaftsordnung}

(1) Wie jede Wirtschaftspolitik, so geht auch die Politik zur Gestaltung der Sozialen Marktwirtschaft von Zielen aus, die im wirtschaftlichen Zusammenleben der Gesellschaft durch Einsatz wirtschaftspolitischer Instrumente (Mittel) realisiert werden sollen. Da es sich bei der Zielbestimmung immer um Wertungen (Werturteile) handeln muß, entsteht sofort die Frage nach dem Subjekt der Wertsetzungen. Die Festlegung der Ziel-(Präferenz-)ordnung durch eine relativ kleine Gruppe von Individuen (z.B. politische Führung eines Landes) nach diktatorischem Prinzip scheidet generell für eine freiheitliche Gesellschaft aus. Subjekte der Zielbestimmungen können hier nur alle Staatsbürger bzw. die von ihnen in demokratischen Wahlverfahren dazu legitimierten Vertreter sein. Wie in der Wohlfahrtstheorie nachgewiesen wurde, ist es dabei nicht möglich, aus den unterschiedlichen Zielen und Interessenlagen der Individuen eine gesellschaftliche Zielfunktion („Wohlfahrtsfunktion“) abzuleiten und als Maßstab der Wirtschaftspolitik zu verwenden. Diese Dilemmasituation bei der gesellschaftlichen Zielbestimmung kann nur pragmatisch im Sinne eines ,second best“ gelöst werden: Nach domokratischen Spielregeln werden diejenigen Grundwerte („oberste“ Ziele) ermittelt, die als gemeinsame Basis von der Mehrheit einer Gesellschaft akzeptiert werden. Im Idealfall werden aus ihnen wirtschafts- und sozialpolitische Ziele widerspruchsfrei abgeleitet, die dann als unmittelbare Richtschnur wirtschaftspolitischer Aktivitäten dienen.

(2) Solche „oberste“ Ziele, auf deren Verwirklichung die Wirtschaftspolitik der Sozialen Marktwirtschaft gerichtet ist, sind Freiheit, Gerechtigkeit und Wohlstand; häufig ergänzt durch die Ziele Frieden und Sicherheit. Ihr Stellenwert für die Ausrichtung der Wirtschaftspolitik in der Sozialen Marktwirtschaft wurde bereits beschrieben. Einige Beispiele sollen den ökonomischen Bezug dieser „obersten“ Ziele sowie die Schwierigkeiten ihrer positiven Definition verdeutlichen: 
a) Freiheit im Sinne des Rechts auf Lebensgestaltung nach individuellen Zielvorstellungen ist nicht vereinbar mit detaillierten Verhaltensvorschriften. Da zur Verwirklichung außerökonomischer Ziele in der Regel knappe Güter notwendig sind, müssen die Menschen über solche Güter verfügen und zwischen Alternativen wählen können. Garantie von Freiheitsrechten setzt also ein solches Wirtschaftssystem voraus, das den Menschen größtmögliche Entscheidungsspielräume im Wirtschaftsprozeß offenhält. Die Grenzen dieser Entscheidungsfreiheit sind durch die gesellschaftlichen Rahmenbedingungen dort zu ziehen, wo die Durchsetzung individueller Interessen zu Lasten der Freiheitsspielräume anderer Gesellschaftsmitglieder geht. Wie schwer es ist, individuelle Entscheidungsspielräume vor politischer und ökonomischer Machtausübung - privater und staatlicher Art - zu schützen, zeigt die gesellschaftliche Realität hinreichend.

b) Der ökonomische Bezug des Zieles der Gerechtigkeit ist ebenfalls evident - denkt man nur an die Verteilung der Produktionsmittel, der produzierten Güter (Sozialprodukt), der Chancen und Risiken im Wirtschaftsprozeß. Das allgemein akzeptierte Gerechtigkeitsziel positiv und operational definieren zu wollen, stößt auf unüberwindbare Schwierigkeiten. Leistungsgerechte, bedarfsgerechte oder völlig gleichmäßige Verteilung der Einkommen auf die Gesellschaftsmitglieder sind denkbare (und in der Diskussion aktuelle) Interpretationen des Gerechtigkeitszieles, die - jeweils mit einem Ausschließlichkeitsanspruch versehen - nicht von allen Mitgliedern der Gesellschaft akzeptiert werden. Wiederum wird nur eine pragmatische Negativabgrenzung dessen weiterhelfen, was allgemein als „unsoziale“ oder „ungerechte“ Verteilung in der Gesellschaft angesehen wird. Die Wirtschaftspolitik in der Sozialen Marktwirtschaft versucht, die Prinzipien leistungs- und bedarfsgerechter Verteilung zu kombinieren.

c) Das Wohlstandsziel in einer Gesellschaft zu verwirklichen, hängt unmittelbar ab von der ökonomischen Leistungsfähigkeit des arbeitsteiligen Wirtschaftsprozesses. Daß es - allgemein akzeptiert - einen hohen Stellenwert in der Rangordnung gesellschafts- und wirtschaftspolitischer Ziele hat, wird einsichtig, wenn man die Existenz von Armut, Elend und sozialen Mißständen im historischen Rückblick bzw. in einzelnen Ländern der Gegenwart betrachtet. Es hat zudem Bezüge zu den beiden erstgenannten Zielen: Mit steigenden Realeinkommen der Wirtschaftssubjekte nehmen die Alternativen der Einkommensverwendung und somit die Entscheidungsmöglichkeiten zu. Gleichzeitig entsteht das Problem der Partizipation des einzelnen am Wohlstand und damit wiederum das der Verteilungsgerechtigkeit.

(3) So wichtig diese „oberste“ Ziele gesellschaftlicher Gestaltung für die Prinzipien der Wirtschaftspolitik der Sozialen Marktwirtschaft sind, bestehen doch erhebliche Probleme, sie als unmittelbares Orientierungsmaß der täglich notwendigen wirtschaftspolitischen Entscheidungen zu verwenden. Der Grund hierfür liegt in ihrer allgemeinen Formulierung und in den Schwierigkeiten, sie 
zu operationalisieren. Aus den negativen Erfahrungen der ökonomischen Entwicklung in der Vergangenheit (z.B. Inflationen seit dem ersten Weltkrieg; Weltwirtschaftskrise 1929-1932) wurden deshalb gesamtwirtschaftliche Ziele abgeleitet, welchen die Rolle ökonomischer Sekundärziele zu den „obersten“ Zielen gesellschaftlichen Zusammenlebens zukommt. An der Realisierung dieser ökonomischen Sekundärziele ist die Wirtschaftspolitik der Sozialen Marktwirtschaft ausgerichtet. Dabei ist stets zu berücksichtigen, daß diese ökonomischen Sekundärziele nie zum Selbstzweck erhoben werden dürfen. Sie dienen lediglich dazu, die abstrakteren übergeordneten Primärziele zu operationalisieren.

\section{b) Gesamtwirtschaftliche Ziele}

(1) In der deutschen Ausgestaltung der Sozialen Marktwirtschaft werden drei zentrale gesamtwirtschaftliche Ziele formuliert: Preisniveaustabilität (Geldwertstabilität), hoher Beschäftigungsstand (Vollbeschäftigung) sowie Zahlungsbilanzausgleich. Das Zielbündel wird wegen des Konkurrenzbzw. Konfliktverhältnisses, in dem die Einzelziele zueinander stehen können, auch als „,magisches Dreieck“ der Wirtschaftspolitik bezeichnet. Begründungen und Inhalte der einzelnen Ziele sollen wegen ihrer Bedeutung für die Wirtschaftspolitik der Sozialen Marktwirtschaft kurz beschrieben werden.

(2) Die Forderung nach Preisniveaustabilität resultiert aus den negativen Erfahrungen, die in Perioden allgemeine Preisanstiegs (Inflation) oder Preisverfalls (Deflation) gemacht wurden. Inflationen begünstigen die Schuldner (Kreditnehmer) und benachteiligen die Gläubiger (Sparer) monetärer Forderungen, da der Realwert von monetären Titeln, die auf eine feste Geldsumme lauten, mit steigenden Preisen proportional sinkt. Der Schuldner zahlt nominal den vereinbarten Betrag zurück und erhält real im Verhältnis zum Preisanstieg einen Gewinn, der Gläubiger erleidet in gleicher Höhe einen Verlust. Umgekehrte Gewinn- und Verlustrechnungen gelten für Deflationen, die allerdings in den vergangenen fünfzig Jahren nicht als Problem der Wirtschaftspolitik anzusehen waren. Inflationsprozesse - und hierbei besonders solche mit steigenden Inflationsraten (,akzelerierte“ Inflation) - treffen besonders die einkommensschwachen Schichten, die sich in der Regel nicht oder kaum in ihrem Verhalten den Inflationsbedingungen anpassen können. Nicht zuletzt können steigende Inflationsraten einen Vertrauensschwund der Wirtschaftssubjekte in die Währung auslösen und damit arbeitsteiliges Wirtschaften gefährden. Die negativen Konsequenzen wurden in der unmittelbaren Nachkriegszeit eindrucksvoll deutlich.

(3) Hoher Beschäftigungsstand - interpretiert als Vollbeschäftigung der Arbeitskräfte in einer Volkswirtschaft - wurde durch die Erfahrungen mit der Massenarbeitslosigkeit in der Weltwirtschaftskrise zum allgemein anerkannten Ziel der Wirtschaftspolitik. Die ökonomischen und sozialen Konsequenzen von Unterbeschäftigung (Arbeitslosigkeit) sind evident: Arbeitslosigkeit bedeutet Verzicht auf an sich mögliche Güterproduktion und insofern Wohlstandsminderung, reduziert die 
Entscheidungsspielräume von Wirtschaftssubjekten, verschärft soziale Konflikte in der Gesellschaft, mindert die Chancen sozialen - und nicht zuletzt politischen - Friedens und ist deshalb mit den „obersten“ Zielen einer freiheitlichen Gesellschaft unvereinbar. Eine absolut „richtige“ Quantifizierung des Vollbeschäftigungszieles gibt es allerdings nicht.

(4) Das Ziel des Zahlungsbilanzausgleichs (,außenwirtschaftliches Gleichgewicht“) bezieht die monetären und realen Außenwirtschaftsbeziehungen in die Wirtschaftspolitik ein. In der Zahlungsbilanz eines Landes werden alle mit dem Ausland getätigkten Transaktionen statistisch für eine Periode (Jahr) erfaßt. Waren- und Dienstleistungsexporte sowie Kapitalimporte führen zu Devisenzuflüssen, Waren- und Dienstleistungsimporte sowie Kapitalexporte zu Devisenabflüssen. Die Zahlungsbilanz gilt als ausgeglichen, wenn in einer Periode der Bestand an ausländischen Währungen eines Landes weder zu- noch abnimmt, zu- und abströmende Devisenmengen also größengleich sind.

(5) Ergänzt man das „magische Dreieck“ noch um das Ziel eines stetigen und angemessenen Wachstums, so kommt man zum „magischen Viereck“. Als Wirtschaftswachstum wird in der Regel der Anstieg des realen Bruttosozialprodukts innerhalb einer Periode (Jahr) verstanden; die Wachstumsrate drückt dann die prozentuale Änderung aus. Interpretiert man Wachstum als Erhöhung des Lebensstandards (Wohlstandsverbesserung), müssen Bevölkerungsentwicklung und Einkommensverteilung berïcksichtigt werden, da z.B. der Lebensstandard bei überproportionalem Bevölkerungswachstum trotz positiver Wachstumsrate sinken kann. Der Zusatz „stetig“ wird in der Regel als Forderung nach gleichmäßigem, nicht in einzelnen Schüben erfolgendem Wachstum interpretiert. Daraus wird die wirtschaftspolitische Aufgabe abgeleitet, zyklische Schwankugen der Wachstumsraten auszuschließen oder wenigstens weitgehend $\mathrm{zu}$ mindern. Unter angemessenem Wachstum wird nicht selten die Realisierung einer möglichst hohen Wachstumsrate verstanden, allerdings unter Berücksichtigung der Wachstumsgrenzen und anderer wirtschaftspolitischer Ziele.

\section{c) Soziale Ziele}

(1) Neben den gesamtwirtschaftlichen Zielen des „magischen Drei- bzw. Vierecks“ sind die bereits erwähnten Ziele sozialer Gerechtigkeit, sozialer Sicherung und sozialen Fortschritts unmittelbar aus der Ordnungsidee der Sozialen Marktwirtschaft ableitbar. Die Verteilung des Sozialprodukts erfolgt in der marktwirtschaftlichen Ordnung nach der einzelwirtschaftlichen Leistung. Leistungsfähige werden nicht, beschränkt Leistungsfähige nur ungenügend durch das Marktprinzip leistungsgerechter Verteilung berücksichtigt. Die hierdurch auftretenden sozialen Härten, die durch das Entstehen von Nicht-Leistungsgewinnen an den Märkten verschäft werden, sind mit der Ordnungskonzeption der Sozialen Marktwirtschaft unvereinbar und bedingen den Einsatz wirtschafts- und sozialpolitischer Instrumente. 
(2) In der Tat: Eine noch so effiziente Marktwirtschaft wird von den Bürgern nicht akzeptiert, wenn die ökonomischen und gesellschaftlichen Konsequenzen des Marktsystems als ungerecht beziehungsweise unsozial empfunden werden. Im Zweifel würde dann die Marktwirtschaft als ganzes abgelehnt. Daher ist es Aufgabe der Politik, die Ergebnisse des Wirtschaftens „gerecht“ zu verteilen und ein soziales Netz für die Bedürftigen zu spannen. Aus einer effizienten und stabilisierten Marktwirtschaft entsteht dann eine Soziale Marktwirtschaft. Unter der Sozialpolitik lassen sich sämtliche Aktivitäten zusammenfassen, die auf soziale Gerechtigkeit und Sicherheit sowie sozialen Friedens abzielen. Hierfür gilt es auch, die materiellen Lebensgrundlagen benachteiligter gesellschaftlicher Gruppen zu verbessern. Das Ziel sozialer Gerechtigkeit wird in der Regel identifiziert mit dem Ziel einer sozial gerechteren Einkommens- und Vermögensverteilung. Für eine exakte Definition dieses Zieles fehlt gegenwärtig allerdings ein aussagefähiger und allgemein akzeptierter Maßstab. Selbst wenn dieser gefunden wäre, bliebe immer noch das Problem zu lösen, bei welchem Wert des Maßstabs dieses Ziel als realisiert anzusehen ist. Das Gerechtigkeitspostulat wird deshalb pragmatisch als Ziel der Verringerung der Einkommensunterschiede in der Gesellschaft interpretiert.

(3) Der Zusammenhang, der zwischen den gesamtwirtschaftlichen und den sozialen Zielen besteht, ist evident: Vermeidung von Arbeitslosigkeit und Inflation ist Voraussetzung für die Verwirklichung sozialer Sicherheit und Gerechtigkeit. Wirtschaftliches Wachstum wiederum ist die Basis sozialen Fortschritts, wenn durch wirtschafts- und sozialpolitische Maßnahmen gesichert ist, daß der ökonomische Fortschritt zur Minderung sozialer Gefälle in der Gesellschaft genutzt wird.

\section{Aufgaben des Staates in der Sozialen Marktwirtschaft: Grundlagen}

\section{a) Die konstituierenden Prinzipien Euckens}

(1) Bei ihren Interventionen muss sich die Wirtschaftspolitik von Prinzipien leiten lassen, die aus dem Grundverständnis der zugrunde liegenden Wirtschaftsordnung abgeleitet sind. Von besonderer Bedeutung sind die von Eucken formulierten „konstituierenden Prinzipien“ einer funktionsfähigen Wettbewerbswirtschaft, deren Beachtung eine zentrale Vorgabe für eine rationale Politik ist:

- Oberstes Prinzip ist das des umfassenden Strebens nach Wettbewerbspreisen; dem liegt die Überzeugung zugrunde, dass das marktwirtschaftliche System allein auf der Grundlage von Wettbewerbspreisen in der Lage ist, seine Lenkungsfunktion wirksam auszuüben.

- Bei dem Prinzip des Primats der Währungspolitik geht es darum, die Stabilität des Preisniveaus zu sichern; dahinter steht die Überlegung, dass inflationäre Preisentwicklungen einer rationalen Wirtschaftsrechnung die Grundlagen entziehen und damit den Lenkungsmechanismus der Marktpreise zerstören. 
- Das Prinzip der Offenen Märkte gewährleistet freien Marktzugang; es ist wesentliche Voraussetzung für die Aufrechterhaltung des Wettbewerbs und die Verhinderung der Entstehung von wirtschaftlichen Machtpositionen.

- Das Prinzip des Vorrangs für das Privateigentum ist die Grundlage für die Zuteilung von Verfügungsrechten.

- Nach dem Prinzip der Vertragsfreiheit steht es den privaten Akteuren frei, mit wem sie Verträge schließen und welchen Inhalt diese haben; das Prinzip gilt allerdings nur mit der Einschränkung, dass diese Freiheit nicht dazu missbraucht werden darf, den Wettbewerb zu beschränken.

- Das Prinzip der Haftung begründet in der auf dezentralen Entscheidungen beruhenden Marktwirtschaft die Verantwortlichkeit derer, die aufgrund der ihnen zugeteilten Verfügungsrechte Entscheidungen treffen.

- Das Prinzip der Konstanz der Wirtschaftspolitik soll mehr Sicherheit für private Investitionsplanungen schaffen, indem es häufige und unvorhersehbare Änderungen der staatlichen Politik ausschließt.

(2) Diese konstitutierenden Prinzipien der Wettbewerbswirtschaft müssen noch um entsprechende konstitutierende Prinzipien für die Gestaltung der Sozialkomponenten ergänzt werden.

\section{b) Ordnungspolitik und/oder Prozesspolitik als Handlungsvorgaben staatlicher Interventionen}

(1) Bei der Umsetzung dieser konstituierenden Prinzipien in konkrete Politik stehen grundsätzlich zwei Wege offen:

* Die zuständigen Instanzen können versuchen, die konkret vorliegende Wirtschaftsordnung direkt zu verändern; in diesem Fall spricht man von ordnungspolitischen Maßnahmen bzw. von Ordnungspolitik.

* Die zuständigen Instanzen können versuchen, durch Eingriffe in den Wirtschaftsablauf bzw. -prozeß, der sich im Rahmen der vorgegebenen Wirtschaftsordnung vollzieht, die Ergebnisse der ökonomischen Aktivitäten zu verändern; in diesem Fall spricht man von ablaufs- oder prozeßpolitischen Maßnahmen bzw. von Ablaufs- oder Prozeßpolitik.

(2) Diese Unterscheidung ist von größter Bedeutung im Gedankengebäude von Eucken. Wirtschaftspolitik soll in erster Linie Ordnungspolitik sein; in ihrem Mittelpunkt soll die Gestaltung der rechtlichen und institutionellen Rahmenbedingungen für das wirtschaftliche Handeln der Unternehmer und der Konsumenten stehen. Ordnungspolitik orientiert sich nicht am Einzelfall; sie ist vielmehr langfristig angelegt und soll gemäß dem Prinzip der Konstanz der Wirtschaftspolitik verlässliche Bedingungen für wirtschaftliches Handeln herstellen. Ordnungskonform (= system- 
konform) sind wirtschaftspolitische Eingriffe aber nur, wenn sie mit der Wirtschaftsordnung kompatibel sind, in deren Rahmen sie erfolgen; sie dürfen also keine Wirkungen auslösen, welche die bestehende Wirtschaftsordnung partiell oder vollständig außer Kraft setzen. Ordnungsinkonform dagegen sind solche wirtschaftspolitische Eingriffe, die sich nicht auf die bloße Durchsetzung allgemeiner gesetzlicher Regeln gründen, sondern notwendig mit willkürlicher Unterscheidung von Personen verbunden sind.

(3) Prozesspolitik besteht im Gegensatz dazu darin, dass der Staat unmittelbar lenkend in das Wirtschaftsgeschehen eingreift, in aller Regel als Reaktion auf konkrete Einzelprobleme; sie zielt dabei auf kurzfristig erreichbare Ergebnisse. Der Prozesspolitik steht Eucken mit großem Misstrauen gegenüber; dies gilt vor allem für staatliche Eingriffe in den Marktmechanismus, also für Preisregulierungen, ebenso aber auch für die Stützung einzelner Branchen und Unternehmen durch Subventionen oder durch Abschirmung gegen ausländische Konkurrenz. Sehr skeptisch beurteilt Eucken auch die Möglichkeiten einer staatlichen Konjunkturpolitik. Er sieht zwar in der Bekämpfung der Arbeitslosigkeit eine Aufgabe von besonderer Bedeutung, bezweifelt aber, dass die Konjunkturpolitik hierfür über die geeigneten Instrumente verfügt. Stattdessen hält Eucken eine Ordnungspolitik, die den Lenkungsmechanismus des Marktes voll zur Geltung kommen lässt, für den besser geeigneten Weg, einen befriedigenden Beschäftigungstand zu erreichen.

(4) Ordnungspolitik bezieht sich nicht nur auf die Wettbewerbsordnung, sie umfasst vielmehr den gesamten Bereich der Wirtschafts- und Sozialpolitik einschließlich des dafür relevanten rechtlichen Rahmens, so insbesondere die Staatsfinanzen, das Geldwesen, die Systeme der sozialen Sicherung und die Regulierung des Arbeitsmarktes. Die für die einzelnen Gebiete geltenden Teilordnungen können nicht unabhängig voneinander gestaltet werden. Eucken weist mit besonderem Nachdruck auf die Interdependenz der Ordnungen hin. Wenn man die Voraussetzungen für eine durch den Marktmechanismus gelenkte Wirtschaft schaffen will, genügt es nicht, für Wettbewerb auf den Märkten zu sorgen; man braucht auch eine Ordnung der Staatsfinanzen, die die Finanzierung staatlicher Aufgaben sicherstellt, ohne private Initiative durch hohe Steuerbelastung zu lähmen, man braucht eine Ordnung für das Geldwesen, die Geldwertstabilität sichert und damit für Investoren die Voraussetzungen für zukunftsgerichtete Wirtschaftsrechnungen schafft. Bei der Gestaltung der Systeme der sozialen Sicherung ist zu beachten, wie die Regelungen sich auf die Funktionsweise des Arbeitsmarktes und damit indirekt auch auf andere Märkte auswirken; das gleiche gilt für Regulierungen des Arbeitsmarkts, die mit sozialpolitischer Begründung zum Schutz der Arbeitnehmer erlassen werden. Die Interdependenz der Ordnungen zwingt dazu, bei allen Regelungen zur Gestaltung der rechtlichen und gesellschaftlichen Gesamtordnung die Konsequenzen für die Funktionsweise der Märkte zu bedenken. Bleibt die Interdependenz der Ordnungen unbeachtet, so können sich Diskrepanzen zwischen den Teilordnungen einstellen, die die Funktionsfähigkeit der Märkte 
beeinträchtigen und schwerwiegende Rückwirkungen für die Leistungsfähigkeit der Wirtschaft, für die Beschäftigung und für den Wohlstand der Bürger eines Landes haben.

\section{Aufgaben des Staates in der Sozialen Marktwirtschaft: Die zentralen Politikfelder}

Nach der Erörterung dieser Grundausrichtung der staatlichen Interventionen in einer Sozialen Marktwirtschaft sollen nun die wichtigsten Politikfelder dargestellt werden, die der Staat „abarbeiten“ muss.

\section{a) Die Schaffung der erforderlichen Rahmenbedingungen}

(1) Voraussetzung für das hinreichende Funktionieren der Sozialen Marktwirtschaft ist es, daß der Staat stabile makroökonomische Rahmenbedingungen garantiert sowie Verhaltensnormen für die Wirtschaftssubjekte definiert und überwacht. Klarheit und Stabilität der Regeln sowie ihre strikte Überwachung sind wichtige Kennzeichen einer marktwirtschaftlichen Ordnung. $\mathrm{Zu}$ den Hauptaufgaben des Staates im ökonomischen Bereich gehört daher, Rechtssicherheit zu schaffen, Vertragsfreiheit zu gewährleisten, für makroökonomische Stabilität zu sorgen und wichtige öffentliche Güter wie Infrastruktur und Ausbildung bereitzustellen.

(2) Vertrauen in die Rechtsordnung kann sich nur bilden, wenn Verhaltensnormen klar definiert sind, landesweit gelten und im Zeitablauf nicht dauernd geändert werden. Deshalb müssen zentrale Verhaltensnormen in Gesetzesform gegossen werden und bei einer autonomen Gerichtsbarkeit einklagbar sein. Ein wesentlicher Aspekt der Rechtssicherheit ist dabei, daß vor dem Gesetz alle gleich sein müssen, einschließlich des Staates und seiner ausführenden Organe. Dies impliziert, daß auch staatliche Stellen vor Gericht beklagt werden können müssen. Eine solche Regelung ist vor allem dann unverzichtbar, wenn private Unternehmen mit Staatsunternehmen konkurrieren sollen. Private Investoren werden solange sehr zurückhaltend sein, wie sie den Eindruck haben, sich gegen behördliche Willkür und einseitige Vorteilsnahme zugunsten von Staatsunternehmen nicht schützen zu können.

(3) Die Flexibilität, mit der sich die Wirtschaft an veränderte Parameter anpassen kann, hängt entscheidend davon ab, ob für die Wirtschaftssubjekte Vertragsfreiheit besteht. Nur wenn jeder nach eigenem Nutzenkalkül mit jedem Verträge schließen kann, ist gewährleistet, daß sich bietende Marktchancen auch optimal genutzt werden. Diese Vertragsfreiheit ist sowohl bei der Produktion und dem Handel von Waren und Dienstleistungen als auch bei der Allokation von Arbeitskräften und von Kapital erforderlich: Unternehmen müssen ihre Produkte selbständig im In- und Ausland vermarkten können. Ebenso müssen sie die Möglichkeit haben, Arbeitskräfte selbständig zu suchen und sich den Zugang zum Kapitalmarkt zu eröffnen. Umgekehrt müssen die privaten Haushalte Zugang zu allen 
Anbietern von Waren und Dienstleistungen sowie zu den Nachfragern nach Arbeit und Kapital haben; es darf m.a.W. keine personen- und/oder firmenspezifischen Restriktionen geben.

(4) Die Planungssicherheit der Unternehmen wird auf mittlere und längere Sicht entscheidend von der makroökonomischen Stabilität der Volkswirtschaft beeinflußt. Zentrale makroökonomische Größen sind das Preisniveau, der Zinssatz und der Wechselkurs. Das Preisniveau wird von der Geldpolitik bestimmt. Deshalb steigt die Inflationsrate, wenn Budgetdefizite über eine Geldmengenexpansion finanziert werden. Aus diesem Grunde sollte die Geldpolitik einzig und allein an der Stabilisierung des Preisniveaus orientiert werden. Dies erfordert aber einen hohen Grad von Autonomie der für die Geldpolitik verantwortlichen Instanzen, also insbesondere der Zentralbank. Eine solche Autonomie kann nur erreicht werden, wenn die Zentralbank auch institutionell von dem für das Staatsbudget zuständigen Finanzministerium getrennt wird. Die Geldmengensteuerung erfolgt über die Kontrolle der Neuemission von Geld, über den Zinssatz und über Mindestreservevorschriften für Banken. Der Zinssatz ist der Indikator für die Knappheit von Kapital in einer Marktwirtschaft. Er hat die doppelte Funktion, die inländische Ersparnis anzuregen und für eine effiziente Verwendung von Kapital zu sorgen. Wie der Zins für das Kapital so ist der Wechselkurs der Knappheitsindikator für ausländische Devisen. Die Wechselkurspolitik muß entsprechend dafür Sorge tragen, daß der Wechselkurs Marktbedingungen entspricht sowie für eine gesamtwirtschaftlich effiziente Verwendung der knappen Devisen sorgt. Es bietet sich hier an, auf staatliche Wechselkursfixierung und Devisenkontrollen zu verzichten und stattdessen einen Devisenmarkt zu entwickeln, der dann zumindest den Unternehmen einen freien Zugang zu Devisen eröffnet.

\section{b) Die Bereitstellung öffentlicher Güter}

(1) Weiterhin muß der Staat bestimmte Dienste ständig bereitstellen, um die wirtschaftliche Entwicklung zu fördern:

* In allen Ländern spielt der Staat in der Ausbildung eine dominierende Rolle, besonders in der Vermittlung der Grundfähigkeiten Lesen, Schreiben und Rechnen, die für eine Vielzahl wirtschaftlicher Aktivitäten unverzichtbar sind;

* in den meisten Ländern stellt der Staat die Infrastruktureinrichtungen für die Wirtschaft (Transport-, Nachrichten- und Energiesysteme) bereit;

* in den meisten Ländern sorgt der Staat für die Versorgung mit wirtschaftlich relevanten Informationen und setzt Normen u.a. für Gewichte, Maße und die Sicherheit am Arbeitsplatz;

* in den entwickelten Volkswirtschaften fördert der Staat die wissenschaftliche und technische Forschung. 
(2) Zwar wird - von Land zu Land in unterschiedlichem Maße - die Durchführung einiger dieser Dienstleistungen häufig staatlichen Unternehmen übertragen; diese sind aber mehr staatliche Hilfsorgane als Unternehmen im eigentlichen Sinn, so daß sich an der obigen Auflistung nichts ändert.

\section{c) Die Wettbewerbspolitik als Politikbereich zur Realisierung der Marktwirtschaftskomponente der Sozialen Marktwirtschaft}

(1) Die Freiheit des Marktes kann dieser nicht selbst, sondern in der Regel nur der Staat herbeiführen und gewährleisten. In diesem speziellen Sinn ist freier Wettbewerb eine „staatliche Veranstaltung“ (W. Eucken) - eine Ausdrucksweise, die ansonsten eher mißverständlich sein dürfte. Deshalb hat der Staat dafür Sorge zu tragen, daß eine gesetzliche Rahmenordnung verwirklicht wird, die zum einen den Marktmechanismus vor Wettbewerbsbeschränkugen schützt und zum anderen mögliche negative Auswirkungen dieses Mechanismus (z.B. durch Machtkonzentrationen) verhindert. Wo die Spielregeln des Wettbewerbs versagen oder zu unerwünschten Ergebnissen führen, soll der Staat intervenieren (z.B. Preisuntergrenzen für Märkte mit anomaler Reaktion des Angebots, Qualitätsauflagen). Als Kriterium solcher Interventionen gilt aber stets die Marktkonformität: Es sind nur solche staatliche Maßnahmen zulässig, die den einzelwirtschaftlichen Bedingungsrahmen ändern, ohne direkt in den Marktprozeß einzugreifen. Staatliche Preisfestsetzungen, Mengenkontingentierungen oder gar generelle Preisstops wären als marktinkonforme Maßnahmen hingegen abzulehnen, weil sie den Marktabstimmungsprozeß außer Kraft setzen. Diese Marktkonformität muß in einem wettbewerblich organisierten Wirtschaftssystem die Maxime aller wirtschaftspolitischen Eingriffe sein; jeder nicht marktkonforme Eingriff - aus welchen Gründen auch immer vollzogen - würde das Funktionieren des Marktmechanismus beeinträchtigen und vermutlich (entsprechend den staatsinterventionistischen Erfahrungen) eine Kette weiterer Eingriffe nach sich ziehen.

(2) Freier Wettbewerb, so wichtig er ordnungspolitisch sein mag, bedeutet dabei keineswegs schrankenlosen Wettbewerb. Wie die Grenzen im Einzelnen zu ziehen sind, ist ein Problem, das sich nicht zeitlos gültig lösen läßt. Bei allen drei Gruppen möglicher Wettbewerbsbeschränkungen gibt es neben ordnungspolitisch unerwünschten auch notwendige Begrenzungen oder Ausschaltungen eines freien Wettbewerbs:

\section{a) Monopole:}

Ein Monopol schließt ex definitione freien Wettbewerb aus. Die nachteiligen Wirkungen eines Monopols nehmen mit seiner Dauer, dem Fehlen von Substitutionsgütern und der davon abhängigen unelastischen Nachfrage zu - Voraussetzungen, die in neuerer Zeit praktisch nur noch für staatliche oder staatlich geschützte Monopole zutreffen. Deshalb herrscht weithin Konsens darüber, daß die Monopolbekämpfung oder die Beseitigung staatlicher Monopole für einen möglichst wirksamen freien Wettbewerb erforderlich ist. Es gibt jedoch Bereiche, in denen ein freier Wettbewerb fehl am Platze wäre (Ausnahmebereiche). Ein „klassischer“ 
Ausnahmebereich ist bspw. das Staatsmonopol für die Papier- und Münzgeldproduktion, die (jedenfalls nach herrschender Meinung) nicht dem Wettbewerb überlassen werden darf.

\section{b) Unlauterer Wettbewerb:}

Freier Wettbewerb ist fairer Wettbewerb, in dem unlautere Methoden unter Rivalen keine Berechtigung haben. Es gibt Praktiken, über deren ordnungspolitischen Unwert kein Streit besteht, z.B. täuschende Reklame, Angestelltenbestechung und Geheimnisverrat. Das Problem besteht jedoch darin, daß eine extensive Ausdehnung des Bereichs „unfairen“ Wettbewerbs den Umfang des freien Wettbewerbs einengt. Freier und fairer Wettbewerb stehen in einem Spannungsverhältnis. Die hohe Bedeutung des freien Wettbewerbs erfordert deshalb eine enge Auslegung des Begriffs der Unlauterkeit.

Bspw. ist nicht einzusehen, wieso eine Preisunterbietung - auch eine Preisunterbietung unter Selbstkosten mit dem Ziel, Rivalen aus dem Rennen zu werfen - ohne weiteres unlauter sein soll, wie oft angenommen wird. Der ökonomisch Stärkere soll sich am Markt durchsetzen und den Schwächeren verdrängen, sofern die Stärke nicht auf einem Monopol beruht oder zu einem dauerhaften Monopol führt.

c) Marktzugangsbeschränkungen:

Nationale und internationale Behinderungen des Marktzugangs waren neben der Existenz von Monopolen für die Klassiker der Anlaß, das Konzept des freien Wettbewerbs zu entwickeln. Ein geschlossener Markt kann wegen seiner wettbewerbsbeschränkenden Wirkungen nicht die ökonomischen Ergebnisse eines offenen, freien Marktes erzielen. Selbst oligopolistische oder monopolistische Anbieter und Nachfrager verhalten sich häufig wie bei freiem Wettbewerb, wenn die Möglichkeit des Zugangs besteht, tatsächlich aber nicht erfolgt (potentieller Wettbewerb). Die Fälle, in denen eine Zugangsbeschränkung wettbewerbspolitisch unstreitig ist, sind sehr selten. Relativ unproblematisch an sich sind staatliche Prüfungen für ganz bestimmte Fähigkeiten (z.B. die eines Arztes) vor Aufnahme einer freien Berufsausübung. Ob dagegen die zahlreichen Eignungs- und Zulassungsprüfungen, die in der Praxis anzutreffen sind, ordnungspolitisch wirksam geboten sind, ist eine ganz andere Frage. Ebenso wenig läßt sich das verbreitete Verbot, eine Lotterie oder eine Spielbank zu betreiben, dadurch rechtfertigen, dass solche Geschäfte der Staat selbst betreibt oder dass er Lizenzen vergibt. Hier wird vielmehr wettbewerbspolitisch, „gesündigt“.

(3) Doch welche Konzeptionen sollten der Wettbewerbspolitik zugrunde liegen? Vom Grundansatz her soll Wettbewerbspolitik dazu beitragen, daß der Marktmechanismus zu optimalen Ergebnissen in Hinblick auf die angestrebten wirtschafts- und gesellschaftspolitischen Ziele führt. Entsprechend ist die Effizienz der Wettbewerbspolitik am Marktergebnis, nicht an der Anzahl von Marktteilnehmern zu messen. 


\section{d) Die Sozialpolitik als Politikbereich zur Realisierung der Sozialkomponente der Sozialen Marktwirtschaft}

(1) Zur Realisierung der Sozialkomponente in der Sozialen Marktwirtschaft ist in erster Linie die Sozialpolitik im weitesten Sinne gefragt. Mit ihrer Hilfe geht es darum, Umverteilungseffekte zugunsten benachteiligter Bevölkerungsgruppen zu bewirken. Solche Umverteilungswirkungen können sowohl monetärer als auch nicht-monetärer Art sein: Monetäre Umverteilungswirkungen ergeben sich bspw. aus einer progressiv gestalteten Einkommensteuer, wenn die Steuereinnahmen zur Finanzierung von Transferzahlungen an einkommensschwache Bevölkerungsgruppen verwendet werden. Nicht-monetäre Wirkungen sind hingegen die Folge, wenn die Steuereinnahmen für Basisgesundheitsdienste oder Primarschulen verwendet werden, die überwiegend den armen Bevölkerungsgruppen zur Verfügung stehen.

(2) Allerdings müssen im Rahmen des Konzepts der Sozialen Marktwirtschaft klare Anforderungsprofile bezüglich der Empfänger solcher Sozialleistungen festgelegt werden. Hierzu muss auf eine wichtige Differenzierung des Begriffes „sozialer Gerechtigkeit“ zurückgegriffen werden, der vielen politischen Diskussionen über Gerechtigkeit häufig übersehen wird:

* Als iustitia distributiva verlangt soziale Gerechtigkeit, dass für jeden Bürger die Voraussetzungen gegeben sind, um überhaupt in die gesellschaftliche Zusammenarbeit investieren zu können. Es geht nicht um Almosen, es geht nicht um Umverteilung, es geht um die Schaffung der Grundvoraussetzungen - etwa durch geeignete Bildungs-, Arbeitsmarkt- und Sozialpolitik -, dass jeder für sich selbst sorgen kann in einer Weise, die auch der Gesellschaft insgesamt zugute kommt. Das ist in jedermanns Interesse.

* Als iustitia commutativa verlangt Gerechtigkeit, dass alle Investoren die berechtigte Erwartung haben können, an den Erträgen ihrer Investition hinreichend zu partizipieren. Eine auf Interventionen und Umverteilungen abzielende Politik führt zum Gegenteil, also zur Ungerechtigkeit, insofern berechtigte Erwartungen nicht erfüllt werden - mit der Folge, dass künftige Investitionen unterbleiben.

(3) Schließlich kommt das „Soziale” der Sozialen Marktwirtschaft auch noch durch die Institutionalisierung von Solidarität zum Ausdruck. Sie beinhaltet die Berücksichtigung all jener, die durch unverschuldete Umstände in Schwierigkeiten geraten - Krankheit, Arbeitslosigkeit, Armut usw. Und eine solche institutionalisierte Solidarität vermag sogar der Ermutigung von Investitionen in die gesellschaftliche Zusammenarbeit dienen:

* Zum einen ist Solidarität - aus ökonomischer Sicht - im Kern eine Versicherung. Jede Investition ist mit Risiken verbunden, und gerade besonders produktive Investitionen, bspw. solche in spezifische Ausbildungen und Fertigkeiten, können mit erheblichen Risiken verbunden sein. Die Gewährleistung, dass man im Verlust- oder Schadensfall nicht ins 
Bodenlose fällt, dass vielmehr die Solidargemeinschaft - durchaus auch durch geeignete private Versicherungssysteme - Auffangmöglichkeiten bietet, diese sozialpolitisch zu erbringende Gewährleistung ist eine wichtige Voraussetzung für produktive, aber riskante Investitionen.

* Zum anderen bedeutet institutionalisierte Solidarität die Etablierung wechselseitiger Abhängigkeiten, die dazu führen, dass jeder etwas zu verlieren hat, und die deshalb die Chancen der Zustimmung eines jeden zu dem System der sozialen Marktwirtschaft verbessern.

Anders gesagt: Eine geeignete Form institutionalisierter Solidarität trägt zum sozialen Frieden bei und erhöht damit die Wahrscheinlichkeit, dass jeder die ihm zustehenden Erträge seiner Investitionen auch ernten kann und nicht befürchten muss, dass solche, die nichts zu verlieren haben - Randalierer, gewaltbereite Demonstranten oder arbeitslose Jugendliche ohne Zukunftsperspektive - diese Erträge gar nicht erst zustande kommen lassen oder vernichten.

(4) Die Grenzen der Sozialpolitik zur Umverteilungspolitik sind dabei fließend. In einem engeren Sinn verfolgt die Umverteilungspolitik das Ziel, die sich als Ergebnis der Marktkräfte einstellende Verteilung von Einkommen und Vermögen im Nachhinein gemäß der herrschenden Gerechtigkeitsvorstellung zu korrigieren. $\mathrm{Zu}$ diesem Zweck können vor allem ein progressives Steuersystem und staatliche Transferzahlungen in Form von Sozialleistungen zum Einsatz kommen.

Bei der Gestaltung einer solchen Politik wird aber häufig übersehen, dass nicht jede Umverteilung sozial ist. In der Tat: Es kann nicht die sozialstaatliche Aufgabe sein, Versorgung für alle zu gewährleisten und jedes denkbare Risiko für jeden denkbaren Personenkreis abzudecken. Das wäre falsch verstandene Solidarität und falsch verstandene Subsidiarität. Natürlich geht es bei der Subsidiarität auch nicht darum, die Gemeinschaft nur noch als Lückenbüßer zu verstehen - darauf hat der Sozialethiker Oswald von Nell-Breuning zu Recht hingewiesen. Richtig verstandene Subsidiarität wendet sich aber gegen ein Übermaß an Staatsinterventionen, die der Entfaltung eigener Selbstverantwortung im Wege stehen. Denn am Ende des Versorgungsstaates, von Ludwig Erhard als „moderner Wahn“ bezeichnet, steht der „soziale Untertan“ und nicht der eigenverantwortliche Bürger.

(5) Das in Deutschland entstandene System der sozialen Sicherung und des Arbeitnehmerschutzes wird von vielen Politikern und Lobbyisten mit den Prinzipien der Sozialen Marktwirtschaft begründet. Für lange Zeit wurde es von Vielen als vorbildlich gepriesen, fand allerdings in einigen seiner Ausprägungen auch von Anfang an Kritik. Es entspricht zwar den Grundgedanken der Sozialen Marktwirtschaft, dass der Marktmechanismus der Ergänzung durch soziale Sicherung und auch einer gewissen Regulierung des Arbeitsmarktes bedarf. Es bleibt jedoch offen, wie die soziale Sicherung und die Marktregulierung im Einzelnen ausgestaltet werden sollen. Es gilt die allgemeine Regel, dass die Interdependenz der Ordnungen zu beachten ist, dass also die Funktionsweise des Marktmechanismus nicht beeinträchtigt werden darf. Die Grenzen einer marktkonformen Gestaltung können jedoch überschritten werden, beispielsweise wenn die Finanzierung der sozialen Sicherung zu einem 
der Wettbewerbsfähigkeit abträglichen Ansteigen der Arbeitskosten führt, wenn der Arbeitsanreiz für Erwerbslose durch Lohnersatzleistungen, die sie auch ohne Arbeit erhalten, wesentlich geschwächt wird, wenn der Kündigungsschutz dazu führt, dass Arbeitgeber sich bei der Einstellung von Personal zurückhalten, wenn die Gewerkschaften bei der Lohnpolitik zu wenig Rücksicht darauf nehmen, dass die Beschäftigung von der Lohnhöhe abhängt, und wenn dadurch Arbeitslosigkeit entsteht.

(6) Nach weitverbreiteter politischer Meinung vieler Autoren, die (noch) auf dem Boden des ursprünglichen Konzepts der Sozialen Marktwirtschaft stehen, sind deren Grundprinzipien vor allem im Bereich der Sozialpolitik mittlerweile weitgehend ausgehöhlt. Als wesentliche Ursache hierfür wird dabei angeführt, dass die Verbindung von marktwirtschaftlicher Freiheit und sozialem Ausgleich zu oft missverstanden wurde. Die „Marktwirtschaft“ und das „Soziale“ werden fälschlicherweise von vielen als etwas Getrenntes, ja Gegensätzliches gesehen. Soziale Gerechtigkeit kann nach dieser Auffassung nur außerhalb des Marktes, also durch staatliche Umverteilung oder durch Einschränkung des Wettbewerbs erreicht werden. Ein solches (fehlerhaftes) Verständnis von Sozialer Marktwirtschaft verkennt zweierlei:

- Erstens: Soziale Ziele können weitgehend gerade durch die freiheitsschützenden und -stärkenden Institutionen des Marktes und des Wettbewerbs erreicht werden. Franz Böhm hat den Wettbewerb einmal „das genialste Entmachtungsinstrument“ genannt. Und nirgendwo gibt es größere Chancen für sozialen Aufstieg durch eigene Leistung als in einer funktionierenden und offenen Marktwirtschaft. Deswegen ist marktwirtschaftliche Ordnungspolitik sehr wohl auch ein wesentliches Stück Sozialpolitik.

- Zweitens: Soziale Marktwirtschaft kann kein schlichtes Umverteilungskonzept sein. Ludwig Erhard hat mit „Wohlstand für alle“ nicht einen Wohlstand gemeint, der uns in den Schoß fällt oder vom Staat zum Null-Tarif vermittelt wird. Gemeint war ein Wohlstand für alle, der durch Leistung gemeinsam erwirtschaftet wird und daher zugleich hohen und steigenden Reallohn sowie ein zunehmendes Maß an sozialer Sicherung bedeuten konnte.

(7) Soziale Sicherung kann also nicht in erster Linie eine Frage der guten Absichten und der sozialpolitischen Details sein. Sie ist in erster Linie eine Frage der Leistungskraft der Volkswirtschaft. Jeder Versuch staatlicher Umverteilung, der die volkswirtschaftliche Leistungskraft überfordert, ist zum Scheitern verurteilt.

\section{Zusammenfassung: Die Soziale Marktwirtschaft als ordnungs- politisches Konzept}

(1) Die Wirtschaftsordnung der „Sozialen Marktwirtschaft“ verbindet auf der Basis der Wettbewerbswirtschaft die freie Initiative mit einem durch die marktwirtschaftliche Leistung gesicherten sozialen Fortschritt. Die Marktkomponente soll sich innerhalb von Rahmenbedingungen 
entfalten, die so konstruiert sind, dass sie weitgehend zu sozial akzeptablen Ergebnissen des Marktes führen. Wirtschaftliche Leistungsfähigkeit gilt wiederum als Voraussetzung dafür, soziale Zielsetzungen $\mathrm{zu}$ realisieren. Insofern wird zwar der Leistungswettbewerb als wirtschaftliche Grundlage sozialer Sicherheit und sozialen Fortschritts gefördert und auch prinzipiell als Regulator der Einkommensverteilung akzeptiert; dem Staat wird jedoch gleichzeitig die Aufgabe einer umfassenden Sozialpolitik zugeschrieben. Wer am Leistungswettbewerb nicht, noch nicht oder nicht mehr teilnehmen und deshalb auch kein Markteinkommen erhalten, kann, soll am ökonomischen Fortschritt partizipieren. Dabei ist dem Subsidiaritätsprinzip Rechnung zu tragen, das der Eigeninitiative des Individuums oder Gruppen von Individuen den Vorzug vor dem Staat gibt.

(2) Als wirtschaftliche Hauptziele der Sozialen Marktwirtschaft wurden in der deutschen Ausgestaltung der Sozialen Marktwirtschaft formuliert: Geldwertstabilität, hoher Beschäftigungsstand sowie Zahlungsbilanzausgleich. Ergänzt man dieses sogenannte „magische Dreieck“ noch um das Ziel eines stetigen und angemessenen Wachstums (Indikator der Erhöhung des Lebensstandards), so kommt man zum „magischen Viereck“.

(3) Neben die gesamtwirtschaftlichen Zielen des „magischen Drei- bzw. Vierecks“ sind die sozialen Ziele sozialer Gerechtigkeit, sozialer Sicherung und sozialen Fortschritts unmittelbar aus der Ordnungsidee der Sozialen Marktwirtschaft ableitbar. Die Verteilung des Sozialprodukts erfolgt in der marktwirtschaftlichen Ordnung nach der einzelwirtschaftlichen Leistung. Leistungsfähige werden nicht, beschränkt Leistungsfähige nur ungenügend durch das Marktprinzip leistungsgerechter Verteilung berücksichtigt. Die hierdurch auftretenden sozialen Härten, die durch das Entstehen von Nicht-Leistungsgewinnen an den Märkten verschärft werden, sind mit der Ordnungskonzeption der Sozialen Marktwirtschaft unvereinbar und bedingen den Einsatz wirtschafts- und sozialpolitischer Instrumente.

(4) Welche Aufgaben des Staates resultieren aus der solchermaßen gekennzeichneten Sozialen Marktwirtschaft? Da der Staat praktisch für die Durchführung wirtschaftspolitischer Maßnahmen verantwortlich ist, die zur besseren Zielerreichung der Sozialen Marktwirtschaft führen, geht es hier faktisch um die wichtigsten Aspekte der Wirtschaftspolitik in der Sozialen Marktwirtschaft. Dabei gilt als Grundforderung, dass es sich bei allen zu ergreifenden Maßnahmen ausschließlich um ordnungskonforme Maßnahmen handeln muss. Ordnungskonform (= systemkonform) sind wirtschaftspolitische Eingriffe aber nur, wenn sie mit der Wirtschaftsordnung kompatibel sind, in deren Rahmen sie erfolgen. Sie dürfen also keine Wirkungen auslösen, welche die bestehende Wirtschaftsordnung partiell oder vollständig außer Kraft setzen. Nicht ordnungskonform dagegen sind solche wirtschaftspolitische Eingriffe, die sich nicht auf die bloße Durchsetzung allgemeiner gesetzlicher Regeln gründen, sondern notwendig mit willkürlicher Unterscheidung von Personen verbunden sind. 
(5) Die politischen Aufgaben des Staates in der Sozialen Marktwirtschaft betreffen im Wesentlichen folgende Handlungsfelder:

a) Schaffung der für den Marktmechanismus erforderlichen Rahmenbedingungen:

Dies umfasst die Sicherstellung stabiler makroökonomischer Rahmenbedingungen sowie die Festlegung und Überwachung von Verhaltensnormen für die Wirtschaftssubjekte. Klarheit und Stabilität dieser Regeln sowie ihre strikte Überwachung sind wichtige Kennzeichen einer marktwirtschaftlichen Ordnung. Zu den Hauptaufgaben des Staates im ökonomischen Bereich gehört daher, Rechtssicherheit zu schaffen, Vertragsfreiheit zu gewährleisten, für makroökonomische Stabilität zu sorgen und wichtige öffentliche Güter wie Infrastruktur und Ausbildung bereitzustellen.

\section{b) Bereitstellung öffentlicher Güter:}

Weiterhin muss der Staat bestimmte Dienste ständig bereitstellen, um die wirtschaftliche Entwicklung zu fördern. Hierzu zählen nach deutschem Verständnis der Bildungsbereich, die Bereitstellung von Infrastruktur (Transport-, Nachrichten- und Energiesysteme), die Versorgung mit wirtschaftlich relevanten Informationen (incl. der Setzung von Normen u.a. für Gewichte, Maße und die Sicherheit am Arbeitsplatz) sowie die Förderung der wissenschaftlichen und technischen Grundlagenforschung. Zwar wird - von Land zu Land in unterschiedlichem Maße - die Durchführung einiger dieser Dienstleistungen häufig staatlichen Unternehmen übertragen; diese sind aber mehr staatliche Hilfsorgane als Unternehmen im eigentlichen Sinn, so dass sich an der obigen Auflistung nichts ändert.

c) Wettbewerbspolitik:

Die Freiheit des Marktes kann dieser nicht selbst, sondern in der Regel nur der Staat herbeiführen und gewährleisten. Deshalb hat der Staat dafür Sorge zu tragen, dass eine gesetzliche Rahmenordnung verwirklicht wird, die zum einen den Marktmechanismus vor Wettbewerbsbeschränkungen schützt und zum anderen mögliche negative Auswirkungen dieses Mechanismus (z.B. durch Machtkonzentrationen) verhindert. Wo die Spielregeln des Wettbewerbs versagen oder zu unerwünschten Ergebnissen führen, soll der Staat intervenieren (z.B. Preisuntergrenzen für Märkte mit anomaler Reaktion des Angebots, Qualitätsauflagen). Als Kriterium solcher Interventionen gilt aber stets die Marktkonformität: Es sind nur solche staatlichen Maßnahmen zulässig, die den einzelwirtschaftlichen Bedingungsrahmen ändern, ohne direkt in den Marktprozess einzugreifen.

\section{d) Sozialpolitik:}

Im Bereich der Sozialpolitik übernimmt der Staat grundsätzlich nachträgliche Korrekturaufgaben. Da die Wachstumspolitik als die beste Sozialpolitik verstanden wird, fällt der staatlichen Sozialpolitik die Korrektur der ursprünglichen Einkommens- und 
Vermögensverteilung zu. Diese erfolgt in Form von Renten, Ausgleichszahlungen, Sozialhilfe, Zuschüssen und Subventionen. Die staatlichen Leistungen sollen jedoch die Leistungsbereitschaft der einzelnen nicht beeinträchtigen. Ergänzend dazu soll der Staat Sozialpolitik zugunsten jener Bevölkerungsgruppen praktizieren, die nicht oder nicht hinreichend (nach bestehenden gesellschaftlichen Wertvorstellungen) am Leistungsprozess partizipieren können. Mit Hilfe der Sozialpolitik sollen Umverteilungseffekte zugunsten benachteiligter Bevölkerungsgruppen bewirkt werden. Solche Umverteilungswirkungen können sowohl monetärer als auch nicht-monetärer Art sein: Monetäre Umverteilungswirkungen ergeben sich bspw. aus einer progressiv gestalteten Einkommensteuer, wenn die Steuereinnahmen zur Finanzierung von Transferzahlungen an einkommensschwache Bevölkerungsgruppen verwendet werden. Nicht-monetäre Wirkungen sind hingegen die Folge, wenn die Steuereinnahmen für Basisgesundheitsdienste oder Primarschulen verwendet werden, die überwiegend den armen Bevölkerungsgruppen zur Verfügung stehen.

(6) Die Soziale Marktwirtschaft deutscher Ausprägung ist, gerade während des immer stärkeren Zusammenwachsens nationaler Märkte, eine gute, leistungsstarke Ordnung. Ihr Versprechen „Wohlstand für alle“ zielt auf Teilhabe möglichst vieler. Dem Staat obliegt es dabei einen sozialen Ausgleich zu schaffen, um die Legitimationsbasis der Marktwirtschaft zu erhöhen. Denn der Leistungswettbewerb sollte durch soziale Absicherung und Unterstützung der Beteiligten, die in diesem Wettbewerb ohne Hilfen nicht mithalten können, ergänzt werden. Dabei geht es darum, Chancengerechtigkeit herzustellen und Bedarfsdeckung minimal zu gewährleisten. Der Leitgedanke ist dabei „Hilfe zur Selbsthilfe“. Allerdings sind der Politik in ihrem Handeln in einer Marktwirtschaft auch Grenzen gesetzt: Die Anreize dürfen nicht durch eine zu starke generelle Umverteilung verzerrt werden. Denn hohe Abgabenlasten sind ein wesentlicher Grund für die lange Wachstumsschwäche Deutschlands gewesen. Dabei hat sich mangelndes Wirtschaftswachstum als die größte Quelle der sozialen Ungerechtigkeit erwiesen, weil die Mittel für solidarisches Verhalten immer knapper wurden. Deshalb ist die Diskussion um mehr oder weniger Staat oberflächlich, denn es geht um bessere Rahmenbedingungen und Regeln. Der Staat muss sich dort zurückziehen, wo er Leistungsmotivation vermindert und sich dort engagieren, wo es um Stärkung der Wachstumskräfte und um Chancengerechtigkeit geht, wie beispielsweise bei der Qualifizierung von Menschen. Vor allem gilt es, Leistungsgerechtigkeit und auch die Generationengerechtigkeit, die bei den Kritikern des Marktes nicht relevant zu sein scheint, nachhaltig zu stärken. Auch in Zeiten der Globalisierung brauchen wir dafür nicht gut gemeinte, sondern gute Politik - nämlich eine Politik, die Ludwig Erhards Modell der Sozialen Marktwirtschaft folgt. 